\title{
Nonequilibrium distribution in quantum dots lasers and influence on laser spectral output
}

\author{
H. Jiang and J. Singh \\ Department of Electrical Engineering and Computer Science, The University of Michigan, \\ Ann Arbor, Michigan 48105
}

(Received 8 October 1998; accepted for publication 2 February 1999)

\begin{abstract}
Self-assembled quantum dot structures used for lasers have shown significant variation in the dot size distribution. In this article, we address the issues related to carrier occupation of these dots as a function of temperature in the absence and presence of lasing. The carrier distributions among different dots are derived in this paper through detailed balance. It is found that at low temperatures the carrier occupation is highly nonequilibrium but with increased temperature it tends towards an equilibrium distribution. Based on this distribution, the threshold current density versus temperature has been calculated. Multimode operation of lasers at different injection levels and temperatures is also examined. The theoretical results are compared with published experimental results. (C) 1999 American Institute of Physics. [S0021-8979(99)03210-7]
\end{abstract}

\section{INTRODUCTION}

Self-assembly effects in strained epitaxy have made it possible to grow high quality semiconductor dot structures. These structures have now made it possible to fabricate quantum dot lasers with reasonably good performance. For example, for the $\mathrm{In}_{0.4} \mathrm{Ga}_{0.6} \mathrm{As} / \mathrm{GaAs}$ quantum dot lasers with modulation bandwidths of $7-8 \mathrm{GHz}$ have been demonstrated. ${ }^{1-3}$ Threshold current values have also been reduced. A number of interesting experimental observations suggest that carrier dynamics and level broadening play very important roles in quantum dot lasers. Some of the key experimental observations may be summarized as follows:

(1) Studies on the temperature dependence of the threshold current show that the temperature dependence is very different from quantum well lasers. The threshold current is not described by a single exponential term with a characteristic temperature. Instead, below $100 \mathrm{~K}$ the threshold current is almost independent of temperature. Above 100 $\mathrm{K}$ the behavior is quite similar to normal semiconductor lasers and is defined by a characteristic temperature $T_{0}$ with a value around $48-85 \mathrm{~K}^{1,2,4}$

(2) It is found that the quantum dot laser usually operates in the multimodes. Only just above threshold current or at high temperature, the single mode operation is observed.

(3) Transmission electron microscopy reveals that there is large size fluctuation of quantum dots. This fluctuation reveals itself in the linewidth of photoluminescence peak. The linewidth [full width at half maximum (FWHM)] of the peak is found to be in the range of $30-60 \mathrm{meV}$.

The temperature insensitivity of the threshold current below $100 \mathrm{~K}$ suggests that carrier distribution in quantum dots at low temperatures is not described by a quasi-equilibrium distribution. This coupled with size fluctuations in the dots plays an important role in threshold current and multimode spectral output. In quantum dot lasers, carriers injected from the contacts enter the active region which consisting of a wetting layer which forms a quantum well region and the quasi-zero-dimensional dots region. Carriers enter the quantum well region where they are captured by the quantum dots. Once in the quantum dots, the carriers can be recombined or be emitted back into the quantum well region. The relative ratio of the capture time, emission back time, and the recombination time is very important in determining the carrier distribution in the dots. Several studies have addressed the issue of carrier distribution in the quantum dots. ${ }^{5-7}$ It is expected that at high temperatures, carriers will reach Fermi distribution. At very low temperature, they will be equally distributed among dots ${ }^{5}$ and carriers in different dots will not be in equilibrium with carriers in other dots. However, there are no studies on how the carrier distribution evolves from very low to high temperatures. In this article, the carrier distribution in quantum dots is derived through detailed balance. The threshold current is calculated numerically with this distribution. Finally, the spectral output of the laser is examined and multimode operation is demonstrated.

\section{THEORETICAL MODEL}

\section{A. Physical model for carrier injection}

Self-assembled quantum dot lasers have an active region that is made up of a quantum well region formed from the wetting layer in the dot formation and the quantum dots themselves. Electrons in the wetting layer are in extended states in the lateral (in the growth plane) but electrons in the dots are confined to the individual dots. The quantum dot laser depends on carrier injection into the dots from $n$ - and $p$-type contacts. The carriers enter the quantum well states from which they are captured into the quantum dots. In Fig. 1, we show a schematic view of the active region of the laser. Electrons are captured into the dot in a time $\tau_{c}$ from the well region. We focus on the electrons since the hole times is expected to be much faster. Once in the dot, the electrons can be recombined with holes in a time $\tau_{s}$ or be emitted to the quantum well in a time $\tau_{e}$. The emission time depends on the electrons absorbing phonon energy and it is expected that 


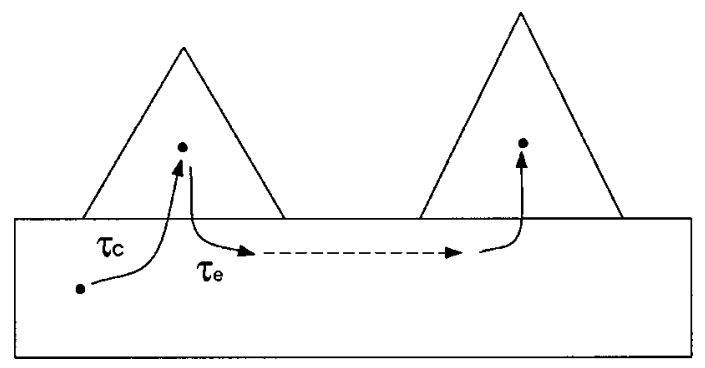

FIG. 1. Transport processes in the quantum dots.

at low temperature this time is very long. If the electrons cannot be emitted back into the well region, the electron density in each dot will be essentially the same regardless of the energy level $E_{i}$ of the discrete state in the dot. However, if the emission time is short (compared to the $e-h$ recombination time), one expects that the carriers will be distributed in a quasi-Fermi distribution among the dots.

We note that each dot can hold a finite number of electrons. Thus, the occupation of each dot is 1 or 2 or 3 , etc. However, it is appropriate to talk of an occupation number which represents an ensemble average for the dot system. This is similar to the use of an occupation number for donor or acceptor occupation.

\section{B. Nonequilibrium Fermi distribution}

As noted above we will focus on electron dynamics since scattering times for holes are much smaller than electrons. Thus the electron dynamics are expected to control device response.

In the case that there is no recombination of electrons and holes, we expect that the system will eventually be in equilibrium and be described by Fermi distribution. Let us assume that there is a single confined level in the quantum dot and the wetting layer is defined by a two-dimensional subband. If the occupation in the wetting layer is low, we can use the Boltzmann distribution for the carrier density in the wetting layer. If $n_{c}$ is this carrier density, we have

$$
n_{c}=N_{c} \exp \left(-\frac{E_{c}-E_{f}}{k_{B} T}\right),
$$

where $E_{c}$ is the wetting layer subband energy. $N_{c}$ is given by

$$
N_{c}=\frac{m_{c}}{\pi \hbar^{2}} k_{B} T
$$

Let us denote the energy level in the dot by $E_{i}$. The probability of occupancy in equilibrium is

$$
f\left(E_{i}\right)=\frac{1}{1+\exp \left[\left(E_{i}-E_{f}\right) / k_{B} T\right]} .
$$

We assume that the quantum dots are uncoupled and tunneling among dots are not important. The equilibrium among dots is thus only reached through the wetting layer. Let us consider the two processes of electron capture into the dots and emission into the wetting layer. Using detailed balance between these two processes, we get for a dot,

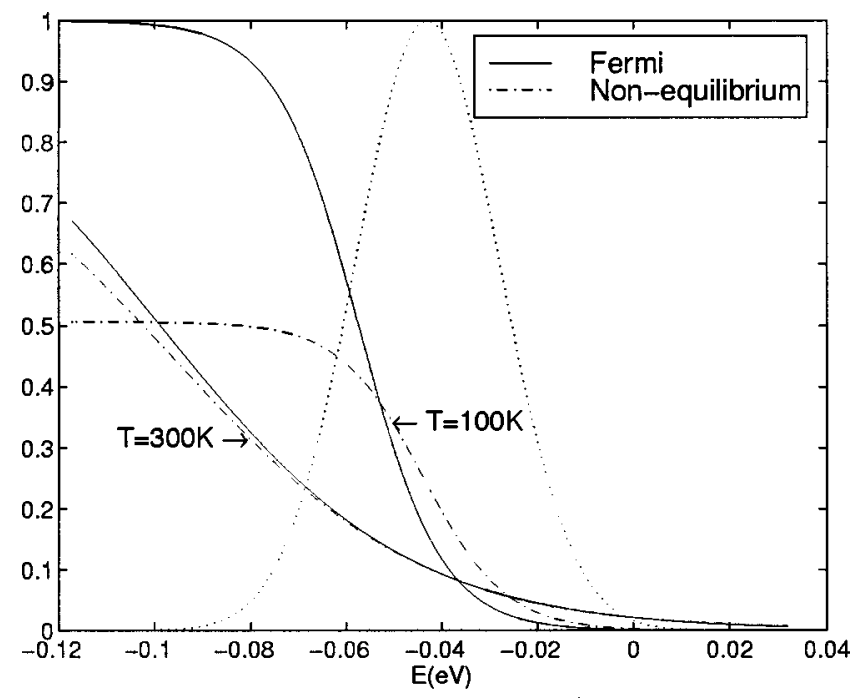

FIG. 2. Standard Fermi distribution and nonequilibrium Fermi distribution. The dotted line is the inhomogeneous broadening function of quantum dots to indicate the size distribution of quantum dots. The parameter $R$ described in the text is $3 \times 10^{-3}$. We choose $E_{c}=0 \mathrm{eV}$. The inhomogeneous broadening parameter $\sigma=15 \mathrm{meV}$.

$$
\frac{n_{c}}{N_{c}}\left(1-f\left(E_{i}\right)\right) \frac{1}{\tau_{c}}=f\left(E_{i}\right) \frac{1}{\tau_{e}},
$$

where $\tau_{c}$ is the capture lifetimes and $\tau_{e}$ is the emission lifetimes. After the equilibrium distribution is put into above equation, we get the relationship between $\tau_{c}$ and $\tau_{e}$ :

$$
\tau_{e}=\tau_{c} \exp \left(\frac{E_{c}-E_{i}}{k_{B} T}\right) .
$$

Let us now consider the recombination process as well. The recombination lifetime is given by $\tau_{s}$. In the steady state, we have to consider the detailed balance between three processes for each dot. This leads to the following equation:

$$
\frac{n_{c}}{N_{c}}\left(1-f\left(E_{i}\right)\right) \frac{1}{\tau_{c}}=\frac{f\left(E_{i}\right)}{\tau_{e}}+\frac{f\left(E_{i}\right)}{\tau_{s}} .
$$

If we define the Fermi level by

$$
n_{c}=N_{c} \exp \left(-\frac{E_{c}-E_{f}}{k_{B} T}\right),
$$

we get the nonequilibrium distribution of the quantum dots: $f\left(E_{i}\right)$

$$
\begin{aligned}
& =\frac{1}{1+\exp \left[\left(E_{i}-E_{f}\right) / k_{B} T\right]+\left(\tau_{c} / \tau_{s}\right) \exp \left[\left(E_{c}-E_{f}\right) / k_{B} T\right]} \\
& =\frac{1}{1+\exp \left[\left(E_{i}-E_{f}\right) / k_{B} T\right]+\left(\tau_{e} / \tau_{s}\right) \exp \left[\left(E_{i}-E_{f}\right) / k_{B} T\right]} .
\end{aligned}
$$

In general, the capture times and recombination lifetimes are different for each dot. However, these differences arising from slightly different sizes of the dots are not expected to be very different. We also expect that the capture time which is controlled by phonon emission processes is not very strongly dependent on temperature.

We see that the occupation distribution is essentially controlled by the ratio 


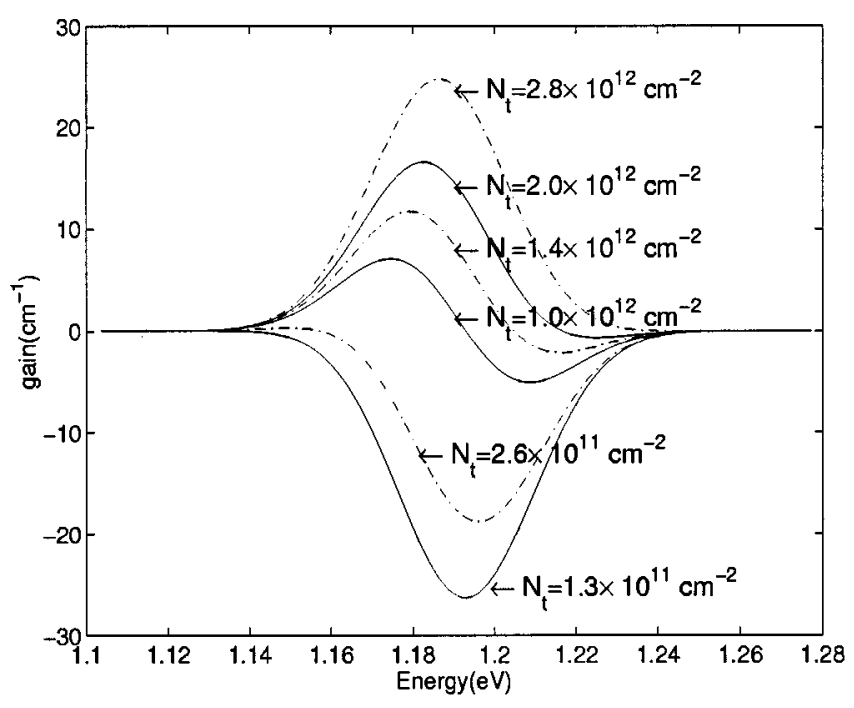

FIG. 3. Gain at room temperature. $N_{t}$ is total carrier density. It is assumed four layers of quantum dots with dot density $5 \times 10^{10} \mathrm{~cm}^{-2}$ in each layer. The width of the optical confinement region, $W$, is chosen to be $0.2 \mu \mathrm{m}$ and the carrier mass in the conduction band is $m_{c}=0.057 m_{0}$.

$$
R \exp \left(\frac{E_{c}-E_{f}}{k_{B} T}\right)
$$

where $R=\tau_{c} / \tau_{s}$. If that ratio is negligible, the distribution is given by the Fermi distribution. However, if that ratio is significant, the distribution has a highly nonequilibrium character. At low temperatures, the value of the ratio is very large since the emission back process depends upon the availability of phonons.

\section{Gain in quantum dots and broadening mechanism}

The cross section of each dot is related to the spontaneous emission by the following relationship:

$$
\sigma_{g}\left(E, E_{i}\right)=\frac{h \lambda^{2}}{8 \pi n^{2}} g_{\text {hom }}\left(E, E_{i}\right) \frac{1}{\tau_{s}}
$$

where $g_{\text {hom }}\left(E, E_{i}\right)$ is the linewidth for that dot with energy level $E_{i}$. There are several contribution to the linewidth of each individual dot: the lifetime of electronic and hole states and the collision from the carriers in the wetting layer and barriers. As done in the atomic laser, those contribution can be described by the Lorentzian broadening function:

$$
g_{\text {hom }}\left(E, E_{i}\right)=\frac{1}{\pi} \frac{\Delta E}{\left(E-E_{i}\right)^{2}+\Delta E^{2}} .
$$

On the other hand, different dot has different size, which will cause inhomogeneous broadening which can be described by Gaussian function:

$$
g_{\text {inh }}\left(E_{i}\right)=\frac{1}{\sqrt{2 \pi} \sigma} \exp \left[-\frac{\left(E_{i}-\bar{E}\right)^{2}}{2 \sigma^{2}}\right] .
$$

In the end, the total gain is

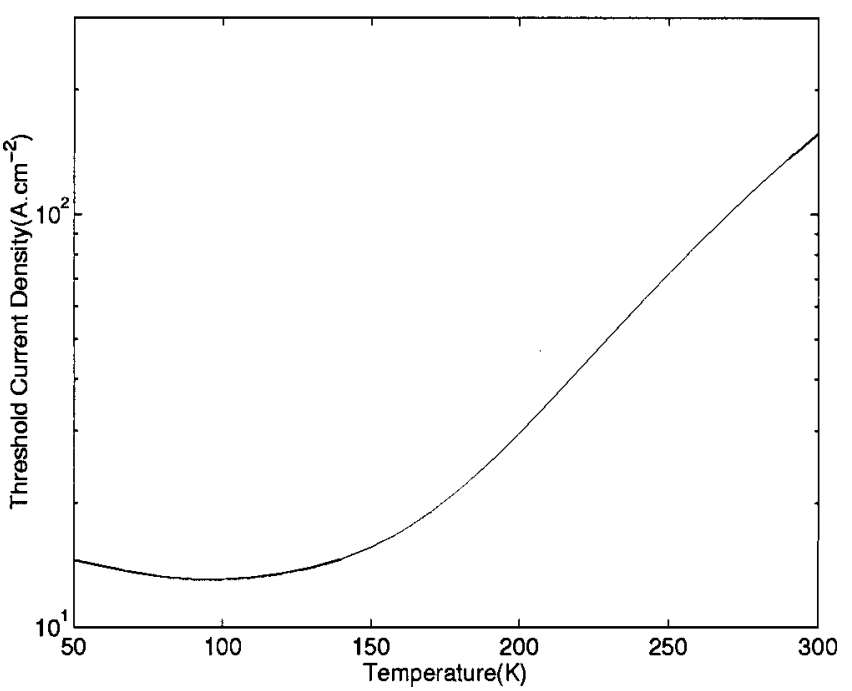

FIG. 4. Threshold current density vs temperature. We take $\tau_{o}=0.6 \mathrm{~ns}, m_{c}$ $=0.057 m_{0}, m_{h}=0.45 m_{0}$. The loss is $20 \mathrm{~cm}^{-2}$. The same quantum dot structure is assumed as Fig. 3 .

$$
\operatorname{Gain}(E)=\int \sigma_{g}\left(E, E_{i}\right) g_{\text {inh }}\left(E_{i}\right) 2 \frac{N_{d}}{W}\left[2 f\left(E_{i}\right)-1\right] d E_{i},
$$

where $N_{d}$ is the dots density and $W$ is the effective width due to optical confinement, the factor 2 is due to spin degeneracy.

In the self-assembled quantum dot case, the inhomogeneous broadening is larger than the homogeneous broadening and the homogeneous broadening can be approximated as $\delta\left(E-E_{i}\right)$. In the end, the gain is

$$
\begin{aligned}
\operatorname{Gain}(E)= & \frac{h \lambda^{2}}{8 \pi n^{2} \tau_{s}} \frac{1}{\sqrt{2 \pi} \sigma} \exp \left[-\frac{(E-\bar{E})^{2}}{2 \sigma^{2}}\right] \\
& \times[2 f(E)-1] 2 \frac{N_{d}}{W} .
\end{aligned}
$$

From the above formula, the maximum gain in selfassembled quantum dots is controlled by the inhomogeneous broadening and dot density.

\section{Carrier distribution at lasing}

When quantum dots are at lasing, the stimulated emission shortens the lifetime of carrier and thus causes stronger nonequilibrium distribution. In general, the laser will operate in multiple modes. The modes are determined by resonant cavity. Suppose there are $n$ modes with photon density $S_{1}$, $S_{2}, \ldots, S_{n}$ in each mode. The carrier distribution is

$$
\begin{aligned}
\frac{d f\left(E_{i}\right)}{d t}= & \frac{n_{c}}{N_{c}}\left[1-f\left(E_{i}\right)\right] \frac{1}{\tau_{c}}-\frac{f\left(E_{i}\right)}{\tau_{e}}-\frac{f\left(E_{i}\right)}{\tau_{s}} \\
& -\frac{c}{n_{g}} \sum_{j} S_{j} \sigma_{g}\left(E_{j}, E_{i}\right)\left[2 f\left(E_{i}\right)-1\right] .
\end{aligned}
$$

The equation for photon density in each mode is 


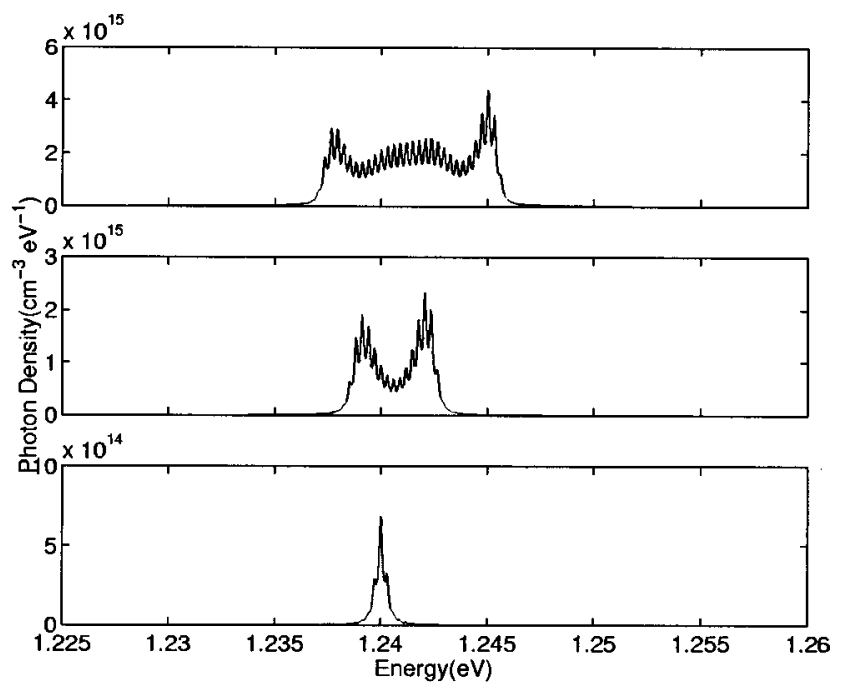

(a)

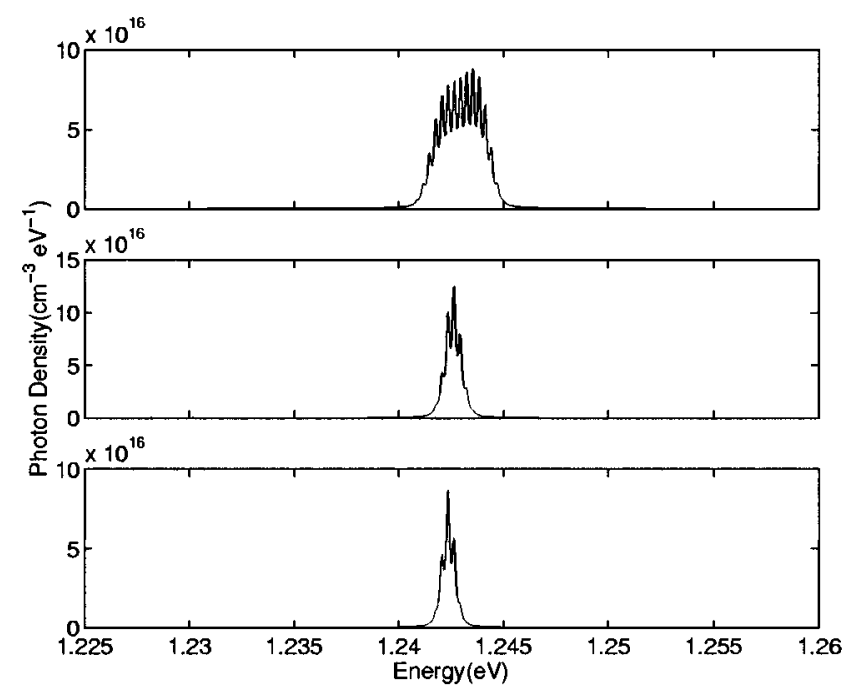

(b)

FIG. 5. Multimodes operation calculated for a self-assembled quantum dot laser. The slope efficiency is assumed to be 0.15 and $\beta=6.5 \times 10^{-9} \mathrm{eV}$. The mode spacing is chosen to be $0.3 \mathrm{eV}$ which corresponds to the $600 \mu \mathrm{m}$ cavity length. (a) $T=100 \mathrm{~K}$. The homogeneous broadening is assumed to be $3 \mathrm{meV}$. The threshold current density is $13 \mathrm{~A} / \mathrm{cm}^{2}$. The injection current density is $14.4,30.5,60.2 \mathrm{~A} / \mathrm{cm}^{2}$ from bottom to top, respectively. (b) $T$ $=300 \mathrm{~K}$. The homogeneous broadening is assumed to be $5.2 \mathrm{meV}$. The threshold current density is $157.2 \mathrm{~A} / \mathrm{cm}^{2}$. The injection current density is $357.7,517.4,872.9 \mathrm{~A} / \mathrm{cm}^{2}$ from bottom to top, respectively.

$$
\frac{d S_{i}}{d t}=\frac{c}{n_{g}}\left[\operatorname{Gain}\left(E_{i}\right)-\gamma\right] S_{i}+2 \beta \frac{N_{d}}{W} \frac{1}{\tau_{s}} f\left(E_{i}\right) g_{\mathrm{inh}}\left(E_{i}\right),
$$

where $\gamma$ is the total loss of the laser cavity, $\beta$ is the spontaneous emission factor. Its unit is $\mathrm{eV}$.

From the above equation, it is easy to see different optical modes coupled together nonlinearly through each quantum dot. The coupling strength among different modes is related to the homogeneous broadening. The larger the homogeneous broadening, the stronger the coupling.

The recombination current density can be calculated as the following:

$$
j=q\left[\frac{n_{d}}{\tau_{s}}+\frac{1}{\tau_{o}} \frac{2 \pi \hbar^{2}}{k_{B} T\left(m_{c}+m_{v}\right)} n_{c}^{2}\right],
$$

where the first term is from the contribution of quantum dots and second term is from the recombination ${ }^{8}$ in the wetting layer. $n_{d}$ is the carrier number in the quantum dots and $n_{c}$ is the carrier number in the wetting layer. Both of them can be calculated after the carrier distribution is known. If we assume the $\tau_{c}$ is a weak function of temperature, the threshold current density can be calculated at different temperature.

\section{RESULTS}

Before discussing our results we need to decide on the values to be used for the position of the dot level, capture time, inhomogeneous and homogeneous broadening, radiative lifetime, etc.

From the photoluminescence (PL) of Ref. 2, the energy difference between wetting layer peak and quantum dot peak is around $50 \mathrm{meV}$. Out of this $50 \mathrm{meV}$, our calculations show that $43 \mathrm{meV}$ is in the conduction band. Thus, in the results shown, $E_{c}-E_{i}$ is chosen to be $43 \mathrm{meV}$ for the average dot. The direct measurement $\tau_{c}$ by pump probe ${ }^{9}$ is around 6 ps. This is the value we use for our calculations. The radiative lifetime is chosen to be $2 \mathrm{~ns}$.

As is the case for an atomic laser, there are two mechanisms for homogeneous broadening in the quantum dot laser. These are lifetime broadening and scattering broadening. We have calculated the scattering rate in the quantum $\operatorname{dot}^{10}$ and have found that the electron hole scattering is strong in this system. In this picture, the hole in the ground states is kicked out at the rate around $0.13 \times 10^{12} \mathrm{~s}^{-1}$. At room temperature, the optical phonon absorption of ground hole states is expected to be as large as $10^{13} \mathrm{~s}^{-1}$. At low temperature, the optical phonon absorption decreases dramatically. The carrier-dot scattering can be estimated as follows: the dimension of dot is $10 \mathrm{~nm}$, the thermal velocity of carriers at room temperature is $10^{7} \mathrm{~cm} / \mathrm{s}$. So the scattering rate is $10 n_{c}$ $=10^{13} \mathrm{~s}^{-1}$, where we use $n_{c}=10^{12} \mathrm{~cm}^{-2}$ at room temperature. These estimates lead to a homogeneous broadening of around $3 \mathrm{meV}$ at room temperature. At low temperature, the carrier-dot scattering dominates the homogeneous broadening.

In Fig. 2, we show the standard Fermi distribution and nonequilibrium Fermi distribution at different temperatures. The solid lines show the Fermi distribution calculated for an injection density of $10^{11} \mathrm{~cm}^{-2}$. The distribution calculated using the formalism given above is shown in the dot-dashed curve.

We see that at $100 \mathrm{~K}$, the actual distribution is highly nonequilibrium. This is because the emission times at low temperatures is very large. With the increase of temperature, the emission times decreases and the system begins to reach equilibrium. We see that at room temperature, the carrier distribution is essentially given by the equilibrium distribution.

In Fig. 3, we show the gain at different injection at 300 $\mathrm{K}$. It is interesting to note there is a small blueshift with increased injection. This arises from the filling of higher energy dot levels at high injection. 
In Fig. 4, the threshold current density versus temperature is shown. At low temperatures, the threshold current decreases a little with increasing temperature. This has been suggested by Ref. 5. As noted above at very low temperatures, the carrier distribution is highly nonequilibrium and the width of the gain curve is very broad. As the temperature increases, the gain width decreases slightly as lower lying dot states arising from larger dot are occupied preferentially. The threshold current density at low temperature is 15 $\mathrm{A} / \mathrm{cm}^{2}$, which falls in the range reported experimentally. We find that the threshold current is more or less independent of temperature up to $\sim 150 \mathrm{~K}$. Beyond this temperature the threshold current starts to increase. The characteristic temperature is $56.5 \mathrm{~K}$ which is in the range reported by several experiments. ${ }^{1,2,4}$ Our results show that the increase of threshold current density at room temperature is due to carrier recombination in the wetting layer.

In Fig. 5, the multimode operation at different injection levels and at different temperatures are shown. When the injected current is just above the threshold current, only three modes are clearly seen. With the increase of the injection current, more modes are observed. Because of the nonlinear interaction between different modes, the intensity distribution does not follow a Gaussian distribution as observed in the photoluminescence. With increasing the temperature, the broadening of modes also decreases as shown by Fig. 5(b).

\section{CONCLUSION}

A nonequilibrium distribution of carrier in the quantum dots are derived. The threshold current, multimode operation are studied in this frame. It shows the general agreement with experiment. Further study for quantum dots with multilevels and lasing behavior at different temperature is in progress.

\section{ACKNOWLEDGMENTS}

The authors gratefully acknowledge supports from NSF (Grant No. ECS9628973) and U.S. Army Research Office.

${ }^{1}$ H. Shoji, Y. Nakata, K. Mukai, Y. Sugiyama, M. Sugawara, N. Yokoyama, and H. Ishikawa, IEEE J. Sel. Top. Quantum Electron. 3, 188 (1997).

${ }^{2}$ D. Bimberg, N. Kirstaedter, N. N. Ledentsov, Zh. I.Alferov, P. S. Kop'ev, and V. M. Ustinov, IEEE J. Sel. Top. Quantum Electron. 3, 196 (1997).

${ }^{3}$ K. Kamath, P. Bhattacharya, T. Sosnowki, T. Norris, and J. Phillips, Electron. Lett. 32, 1374 (1996).

${ }^{4}$ D. J. Klotzkin, Ph.D. dissertation, University of Michigan, 1998.

${ }^{5}$ M. Grundmann and D. Bimberg, Jpn. J. Appl. Phys., Part 1 36, 4181 (1997).

${ }^{6}$ M. Grundmann and D. Bimberg, Phys. Rev. B 55, 9740 (1997).

${ }^{7}$ K. Mukai, N. Ohtsuka, H. Shoji, and M. Sugawara, Appl. Phys. Lett. 68, 3013 (1996)

${ }^{8}$ J. Singh, Semiconductor Optoelectronics (McGraw-Hill, New York, 1995), Chap. 4.

${ }^{9}$ T. S. Sosnowski, T. B. Norris, H. Jiang, J. Singh, K. Kamath, and P. Bhattacharya, Phys. Rev. B 57, R9423 (1998).

${ }^{10}$ H. Jiang and J. Singh, IEEE J. Quantum Electron. 34, 1188 (1998). 